\title{
On the Quantum Transport through an Asymmetric Aharonov-Bohm Ring with Rashba Spin-Orbit Interaction
}

\author{
D.M. BALTATEANU* \\ West University of Timişoara, Faculty of Physics, Bd. V. Pârvan, No. 4, RO-300223, Timişoara, Romania
}

(Received October 17, 2010; in final form July 28, 2011)

\begin{abstract}
The transmission amplitude through an asymmetric Aharonov-Bohm ring is derived analytically, in the presence of the Rashba spin-orbit interaction. For this purpose, one resorts to the transfer matrix method. The conductance is established in terms of the Landauer formalism and it is analyzed for different values of the asymmetry and ring-lead coupling parameters. A theoretical proposal to use the asymmetric ring like a spin filtering device is discussed, by an adequate tuning of the Aharonov-Bohm and Aharonov-Casher phases (via the magnetic field and the Rashba parameter).
\end{abstract}

PACS: 73.23.-b, 71.70.Ej, 73.23.Ad

\section{Introduction}

The spin dependent transport through mesoscopic systems has become a very interesting topic in the last years. There has been a growing interest in the so-called spintronic devices that are based on the spin properties of the electrons. In this new field of research, the major goal is the coherent transport and manipulation of spin in mesoscopic semiconductor devices $[1,2]$. A fundamental concept in semiconductor spintronics is the control of spin through spin-orbit interaction (SOI), where the orbital motion of electrons is coupled with the orientation of electron spins [3]. There are two types of SOI which are relevant for semiconductor spintronics: the Rashba SOI, induced by the asymmetry associated with the confining potential in the growth direction [4] and the Dresselhaus SOI, which is a result of the inversion asymmetry of the bulk crystal [5]. The Rashba coupling becomes important in semiconductors with a small band gap [6] and the coupling parameter can be tuned by an external gate voltage $[7,8]$. The Dresselhaus SOI dominates in the semiconductors with a wide band gap [9] while the related coupling is a material constant.

An important mesoscopic device for the study of the quantum interference phenomena is the quantum ring. The Aharonov-Bohm (AB) [10] and Aharonov-Casher (AC) [11] effects are typical manifestations of quantum interference in such type of structures. In a coherent ring threaded by external magnetic flux the wave function of the electron acquires an $\mathrm{AB}$ phase. In the presence of the Rashba SOI, the so-called AC phase has also to be accounted for.

\footnotetext{
* e-mail: bdmarcel@yahoo.com
}

The spin-dependent properties of quantum rings with the Rashba coupling were studied in many papers [12-17], symmetric rings being utilized in most cases. On the other hand, recently there has been a growing interest for asymmetric configurations, in which the asymmetry has a great influence on the electron transport [18-24].

Some of the theoretical papers in this area [20-23] are focused on the investigation of the transport properties for asymmetric rings subject to magnetic flux, using the standard transfer matrix formalism [25]. Motivated by these works, we will study in this paper the case in which a one-dimensional and asymmetric ring (with unequal lengths of the arms) is subject to both magnetic flux and the Rashba SOI, considering different coupling parameters between the leads and the ring. Other details concerning the transport through asymmetric $\mathrm{AB}$ rings in the presence of the Rashba SOI have also been discussed [26], now with a special emphasis on the role of a delta-barrier.

The paper is organized as follows: In Sect. 2, the one-electron problem for the asymmetric $\mathrm{AB}$ ring in presence of the Rashba SOI is given, according to Ref. [15]. At the end of this section, the transmission amplitude is analytically derived using the transfer matrix formalism [25] and the conductance is obtained, in the Landauer formalism [27]. In Sect. 3, the influence of the AB magnetic flux and the Rashba SOI on the conductance of the asymmetric $\mathrm{AB}$ ring is investigated numerically and the concluding remarks are given in Sect. 4 .

\section{Formalism}

\subsection{The one-electron Hamiltonian and energy spectrum}

We consider a one-dimensional $\mathrm{AB}$ ring of radius $R$ in the $x-y$ plane, asymmetrically coupled to two semi-infinite leads, in the presence of electric and magnetic 
fields (Fig. 1a). The electric field $\boldsymbol{E}_{R}$ is time independent and originates from an asymmetric confinement along the $z$-direction.

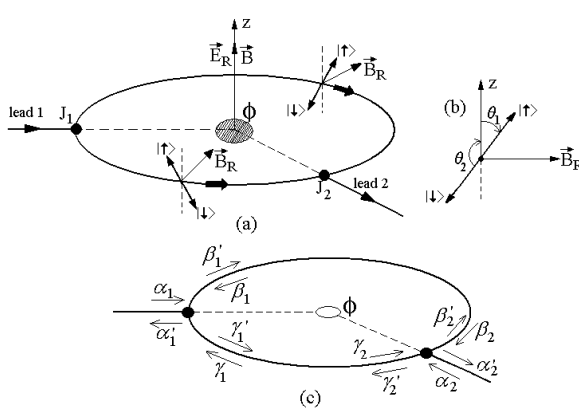

Fig. 1. (a) AB asymmetric ring with Rashba SOI; the electric field $\boldsymbol{E}_{R}$ is perpendicular on the plane of the ring; the magnetic field $\boldsymbol{B}_{R}$ is in the radial direction and its sense depends on the movement directions (clockwise or counterclockwise). (b) Tilt angle $\theta_{1}\left(\theta_{2}\right)$ for the up (down) states. (c) Incoming and outgoing amplitudes at the junctions of the ring.

In the reference frame of the moving electron, a magnetic field $\boldsymbol{B}_{R}$ is generated, which is perpendicular to the electric field and to the movement direction. The external (and time independent) magnetic field $\boldsymbol{B}=\nabla \times \boldsymbol{A}$ is pointing in the $z$-direction, where the vector potential $\boldsymbol{A}$ has the components $(0,0, \Phi / 2 \pi R)$ in cylindrical coordinates. The AB magnetic flux piercing the ring is denoted by $\Phi$.

The 2D one-electron Hamiltonian in the presence of the magnetic field and Rashba SOI reads [14]:

$$
\begin{aligned}
H & =\frac{1}{2 m^{*}}(\boldsymbol{p}-e \boldsymbol{A})^{2}+\frac{\alpha_{R}}{\hbar}[\boldsymbol{\sigma} \times(\boldsymbol{p}-e \boldsymbol{A})]_{z} \\
& -\mu \boldsymbol{B} \cdot \boldsymbol{\sigma}+V(\boldsymbol{r}) .
\end{aligned}
$$

Here, $\alpha_{R}$ is the Rashba coupling constant, $\sigma=\left(\sigma_{x}, \sigma_{y}\right.$, $\left.\sigma_{z}\right)$ are the spin Pauli matrices, $\mu$ is the Zeeman coupling constant for the electron and $V(\boldsymbol{r})$ is the confining potential, whereas the effective mass is denoted by $m^{*}$.

For a weak magnetic field, the Zeeeman term can be neglected and, in the limit of a very narrow ring, the 1D dimensionless Hamiltonian reads [15, 28]:

$$
H=\left(-\mathrm{i} \frac{\partial}{\partial \varphi}+\frac{\xi}{2} \sigma_{r}-\frac{\Phi}{\Phi_{0}}\right)^{2},
$$

where $\varphi$ is the polar angle, $\xi=2 m^{*} R \alpha_{R} / \hbar^{2}$ is the dimensionless spin-orbit coupling strength, $\sigma_{r}=\sigma_{x} \cos \varphi+$ $\sigma_{y} \sin \varphi$ and $\Phi_{0}=h / e$ is the flux quantum.

The energy spectrum for the Hamiltonian in Eq. (2) reads [15]:

$$
E_{n}^{\sigma}=\left(n-\frac{\Phi_{\mathrm{AC}}^{\sigma}}{2 \pi}-\frac{\Phi_{\mathrm{AB}}}{2 \pi}\right)^{2}
$$

where $n \in \mathbb{Z}$ is the orbital quantum number, $\sigma$ is the spin index $(\sigma=1(\uparrow)$ for the spin-up and $\sigma=2(\downarrow)$ for the spin-down), $\Phi_{\mathrm{AB}}$ is the $\mathrm{AB}$ phase acquired by the charged particle in the quantum ring [15]:

$$
\Phi_{\mathrm{AB}}=2 \pi \frac{\Phi}{\Phi_{0}}
$$

and $\Phi_{\mathrm{AC}}^{\sigma}$ is the AC phase [15]:

$$
\Phi_{\mathrm{AC}}^{\sigma}=-\pi\left(1+(-1)^{\sigma} \sqrt{\xi^{2}+1}\right) .
$$

The corresponding eigenstates are given by

$$
\psi_{n}^{\sigma}(\varphi)=\exp (\mathrm{i} n \varphi) \times \chi_{n}^{\sigma}(\varphi),
$$

where $\chi_{n}^{\sigma}(\varphi)$ are orthogonal spinors. These spinors can be expressed in terms of the eigenvectors $(1,0)^{\mathrm{T}},(0,1)^{\mathrm{T}}$ of the Pauli matrix $\sigma_{z}$ as

$$
\begin{aligned}
& \chi_{n}^{(1)}(\varphi)=\frac{1}{\sqrt{2 \pi}}\left[\cos \frac{\theta_{1}}{2}, \exp (\mathrm{i} \varphi) \times \sin \frac{\theta_{1}}{2}\right]^{\mathrm{T}}, \\
& \chi_{n}^{(2)}(\varphi)=\frac{1}{\sqrt{2 \pi}}\left[\cos \frac{\theta_{2}}{2},-\exp (\mathrm{i} \varphi) \times \sin \frac{\theta_{2}}{2}\right]^{\mathrm{T}},
\end{aligned}
$$

where $\mathrm{T}$ stands for the transpose of the matrix and $\theta_{1}\left(\theta_{2}\right)$ is the spin-up (spin-down) tilt angle relative to the $z$ direction.

For the spin-up and spin-down eigenstates, the tilt angle is given by the relations $\tan \left(\theta_{1}\right)=\xi$ and $\theta_{2}=\pi-\theta_{1}$, respectively (Fig. 1b). For a ring which is not connected to leads, the quantum number $n$ is integer, due to the periodicity condition

$$
\psi_{n}^{\sigma}(\varphi)=\psi_{n}^{\sigma}(\varphi+2 \pi m), m \in \mathbb{Z} .
$$

When the ring is coupled to leads, this boundary condition is altered and the quantum number $n$ may be any real number allowed by the energy of the electron.

There are two limit cases i.e. the strong coupling (adiabatic regime) when $\xi \gg 1$ and the weak coupling (non-adiabatic regime) when $\xi \ll 1$ [14]. In the adiabatic limit, the spin eigenstates follow the local direction of the magnetic field $\boldsymbol{B}_{R}$, the spins being aligned parallel or antiparallel to the magnetic field.

\subsection{The transmission amplitude of the asymmetric $A B$ ring}

The $\mathrm{AB}$ ring illustrated in Fig. 1a is asymmetrically coupled to the external leads at the quantum point contacts $J_{1}$ and $J_{2}$. The motion of the electron through this ring is ballistic and thus the spin-flip processes are neglected. In addition, the adiabatic regime is considered, in which the electrons with spin-up and spin-down move independently through the ring (transitions between the two spin channels are not possible).

In this situation, we can obtain analytically the transmission amplitude and the conductance, using the transfer matrix method, with the scattering matrix of the form [25]:

$$
S=\left(\begin{array}{ccc}
-(a+b) & \sqrt{\varepsilon} & \sqrt{\varepsilon} \\
\sqrt{\varepsilon} & a & b \\
\sqrt{\varepsilon} & b & a
\end{array}\right),
$$

where $a= \pm\left((1-2 \varepsilon)^{1 / 2}-1\right) / 2$ and $b= \pm\left((1-2 \varepsilon)^{1 / 2}+\right.$ 
1)/2 with $0 \leq \varepsilon \leq 1 / 2, \varepsilon$ being the coupling parameter $(\varepsilon=1 / 2$ corresponds to fully transparent contacts and $\varepsilon=0$ corresponds to totally reflective contacts).

We consider an incident wave (of unit amplitude, $\alpha_{1}=$ 1) that comes from the left lead with fixed Fermi energy, propagates coherently through the ring branches and it is transmitted to the right lead. It can be mentioned that when the Rashba SOI is taken into account, the electrons in the ring have different wave numbers, depending on the direction of propagation (clockwise or counterclockwise) and on spin orientation $(\sigma=1,2)$.

The wave amplitudes in the upper (lower) arm of the asymmetric $\mathrm{AB}$ ring (Fig. 1c) are inter-related according to [25]:

$$
\left(\begin{array}{c}
\beta_{2} \\
\beta_{2}^{\prime}
\end{array}\right)=\underline{t}_{\mathrm{I}}^{\sigma}\left(\begin{array}{c}
\beta_{1}^{\prime} \\
\beta_{1}
\end{array}\right) ; \quad\left(\begin{array}{c}
\gamma_{1} \\
\gamma_{1}^{\prime}
\end{array}\right)=\underline{t}_{\mathrm{II}}^{\sigma}\left(\begin{array}{c}
\gamma_{2}^{\prime} \\
\gamma_{2}
\end{array}\right),
$$

where $t_{\mathrm{I}}^{\sigma}\left(t_{\mathrm{II}}^{\sigma}\right)$ denotes the transfer matrix for the upper (lower) arm.

For ballistic transport through the arms of the asymmetric $\mathrm{AB}$ ring, the transfer matrices read

$$
\begin{aligned}
& \underline{t}_{\mathrm{I}}^{\sigma}=\exp \left(-\mathrm{i} \chi_{\mathrm{u}}^{\sigma}\right) \times\left(\begin{array}{cc}
\exp \left(\mathrm{i} \varphi_{\mathrm{u}}\right) & 0 \\
0 & \exp \left(-\mathrm{i} \varphi_{\mathrm{u}}\right)
\end{array}\right), \\
& \underline{t}_{\mathrm{II}}^{\sigma}=\exp \left(-\mathrm{i} \chi_{\mathrm{d}}^{\sigma}\right) \times\left(\begin{array}{cc}
\exp \left(\mathrm{i} \varphi_{\mathrm{d}}\right) & 0 \\
0 & \exp \left(-\mathrm{i} \varphi_{\mathrm{d}}\right)
\end{array}\right) .
\end{aligned}
$$

Here $\varphi_{\mathrm{u}}\left(\varphi_{\mathrm{d}}\right)$ is the dynamic phase acquired by an electron with spin-up or spin-down traversing the upper (lower) arm in the clockwise direction. $\chi_{\mathrm{u}}^{\sigma}\left(\chi_{\mathrm{d}}^{\sigma}\right)$ is the phase acquired by the electron with spin-up or spin-down in the upper (lower) arm in the presence of the AB flux and Rashba SOI.

An asymmetry factor like $F=\left(1-F_{a}\right) /\left(\left(1+F_{a}\right)\right.$, where $F_{a}=L_{\mathrm{d}} / L_{\mathrm{u}}$, in which $L_{\mathrm{d}}\left(L_{\mathrm{u}}\right)$ is the length of the lower (upper) arm, can also be introduced [29]. Due to the unequal lengths of the ring arms, the phases $\varphi_{\mathrm{u}}$ and $\varphi_{\mathrm{d}}$ are different

$$
\begin{aligned}
& \varphi_{\mathrm{u}}=k L_{\mathrm{u}}=k \pi R(1+F), \\
& \varphi_{\mathrm{d}}=k L_{\mathrm{d}}=k \pi R(1-F) .
\end{aligned}
$$

The phases $\chi_{\mathrm{u}}^{\sigma}$ and $\chi_{\mathrm{d}}^{\sigma}$ are then given by

$$
\begin{aligned}
& \chi_{\mathrm{u}}^{\sigma}=\left(\Phi_{\mathrm{AB}}+\Phi_{\mathrm{AC}}^{\sigma}\right) \frac{1+F}{2}, \\
& \chi_{\mathrm{d}}^{\sigma}=\left(\Phi_{\mathrm{AB}}+\Phi_{\mathrm{AC}}^{\sigma}\right) \frac{1-F}{2} .
\end{aligned}
$$

Assuming that $\alpha_{2}=0$, the transmission amplitude in the right lead is given by [25]:

$$
T^{\sigma}=\alpha_{2}^{\prime}=-\frac{\varepsilon}{b^{2}}(b-a, 1) \underline{t}_{\mathrm{I}}^{\sigma}\left(\underline{M}^{\sigma}\right)^{-1}\left(\begin{array}{c}
b-a \\
-1
\end{array}\right),
$$

with

$$
\begin{aligned}
& \underline{M}^{\sigma}=\underline{t}_{\mathrm{L}} \underline{t}_{\mathrm{II}}^{\sigma} \underline{t}_{\mathrm{L}} \underline{t}_{\mathrm{I}}^{\sigma}-\left(\begin{array}{ll}
1 & 0 \\
0 & 1
\end{array}\right), \\
& \underline{t}_{\mathrm{L}}=\frac{1}{b}\left(\begin{array}{cc}
b^{2}-a^{2} & a \\
-a & 1
\end{array}\right) .
\end{aligned}
$$

From Eqs. (18), (19) and (20) the analytical expression for the transmission amplitude results

$$
\begin{array}{r}
T^{\sigma}(\eta, \xi, F)=\mathrm{i} \varepsilon\left[\exp \left(\mathrm{i} \frac{\Phi^{\sigma}}{2}(1-F)\right) \sin \left(\frac{\eta}{2}(1+F)\right)\right. \\
\left.+\exp \left(-\mathrm{i} \frac{\Phi^{\sigma}}{2}(1+F)\right) \sin \left(\frac{\eta}{2}(1-F)\right)\right] \\
/\left[a^{2} \cos (\eta F)+b^{2} \cos \left(\Phi^{\sigma}\right)+(\varepsilon-1) \cos (\eta)+\mathrm{i} \varepsilon \sin (\eta)\right],
\end{array}
$$

where $\Phi^{\sigma}=\Phi_{\mathrm{AB}}+\Phi_{\mathrm{AC}}^{\sigma}$ and $\eta=2 \pi k R$.

For the coupling parameter $\varepsilon=4 / 9$ (strong but not maximal ring-lead coupling), the transmission amplitude coincides with those from Ref. [29], for $\Phi_{\mathrm{AB}}=0$. This value for $\varepsilon$ corresponds to the approach of the quantum waveguide theory, in which the (spin-dependent) Griffith boundary conditions are used for obtaining the transmission amplitude [30].

If $\varepsilon=4 / 9$ and $F=0$ (symmetric ring), one has

$$
T^{\sigma}(\eta, \xi, F)=\frac{8 \mathrm{i} \cos \left(\Phi^{\sigma} / 2\right) \sin (\eta / 2)}{1-5 \cos (\eta)+4 \cos \left(\Phi^{\sigma}\right)+4 \mathrm{i} \sin (\eta)},
$$

which reproduces the result done before [15] if $\Phi_{\mathrm{AB}}=0$.

The conductance of the mesoscopic system, in the Landauer formalism, is given by [27]:

$$
G=\frac{e^{2}}{h} \sum_{\sigma=\uparrow, \downarrow}\left|T^{\sigma}\right|^{2}=G^{\uparrow}+G^{\downarrow},
$$

which will be applied hereafter.

\section{Numerical results and further details}

$$
\text { 3.1. Free system }\left(\Phi^{\sigma}=0\right)
$$

As a first step, the behaviour of the conductance in a free system function of the asymmetry parameter and the coupling strength will be analysed. Concerning the asymmetry, an important aspect is that for a certain coupling limit, a very small difference between the ring branches leads to the appearance of the periodical conductance zeros, as a consequence of the asymmetry-dependent interference conditions.

In Fig. 2 the dimensionless conductance function of $\eta$ is represented, for a maximal coupling between the leads and ring and for different and very small values of the asymmetry parameter. For a symmetric ring $(F=0)$, the absence of the backscattering effects leads to a perfect transmission and the conductance is a constant function (straight solid line in Fig. 2). A very small ring asymmetry, $F=0.0256$ (corresponding to $L_{\mathrm{d}}=0.95 L_{\mathrm{u}}$ ), induces a change in the transmission probability and periodical conductance zeros appear (dashed line in Fig. 2). By increasing the difference in length between the ring arms 
( $F=0.0526$ or $\left.L_{\mathrm{d}}=0.9 L_{\mathrm{u}}\right)$, the conductance asymmetry is enhanced (solid line in Fig. 2).

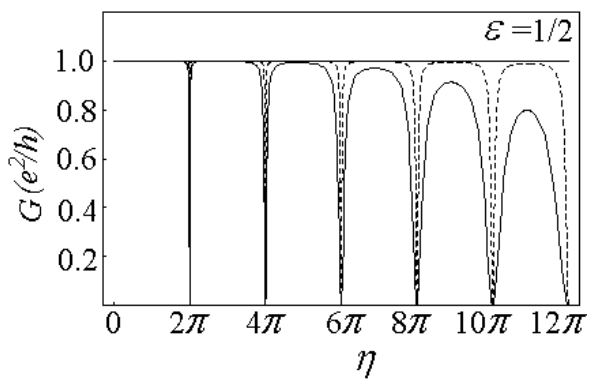

Fig. 2. The dimensionless conductance function of $\eta$, in the case of strong coupling limit $(\varepsilon=1 / 2)$, for $F=0$ (straight line), $F=0.0256$ (dashed line) and $F=0.0526$ (solid line).

If the coupling between the leads and ring is decreased, the imperfect transmission (as a consequence of backscattering effects) leads to appearance of the periodical conductance oscillations [25]. These oscillations are displayed in Fig. 3 for a symmetric $(F=0)$ configuration (dashed curves) and for an asymmetric configuration with the asymmetry parameter $F=1 / 3$ (solid curves), at two different values of the coupling strength.

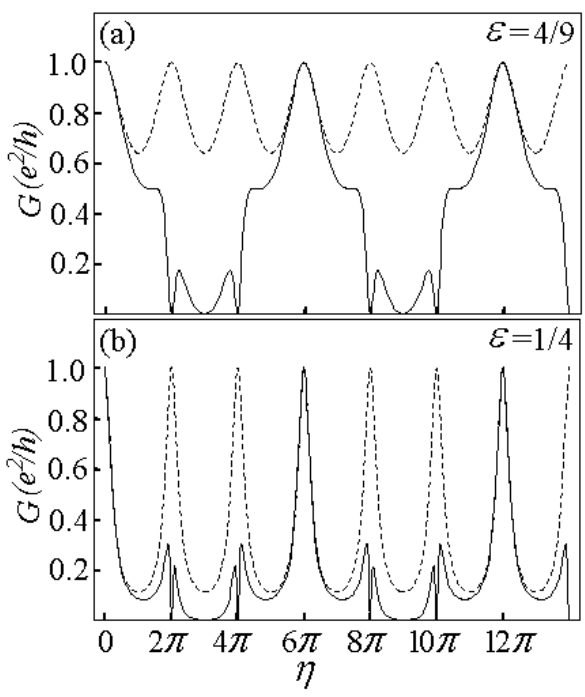

Fig. 3. The dimensionless conductance as function of $\eta$ for different values of the coupling strength: (a) $\varepsilon=4 / 9$; (b) $\varepsilon=1 / 4$. The dashed line corresponds to the symmetric ring $(F=0)$ and the solid line to an asymmetric ring with $L_{\mathrm{d}}=0.5 L_{\mathrm{u}}(F=1 / 3)$.

In the free ring different resonant states appear, due to the time reversal symmetry and the zero conductance resonances (antiresonances) are a manifestation of the destructive interference of such resonant states. It must be pointed out that the antiresonances are characterized by a typical zero-pole structure in the complex energy plane $[29,31]$.
For the symmetric ring (Fig. 3) the period of the conductance oscillation is $2 \pi$ and there are no conductance zeros (antiresonances) due to the compensation of all zeros of the transmission amplitude with the corresponding poles. For asymmetric ring, the period of conductance oscillations strongly depends on the asymmetry parameter $F$.

One has to realize that, in general, the ratio between the lower and upper arms of the asymmetric ring is of the form $L_{\mathrm{d}} / L_{\mathrm{u}}=l_{1} / l_{2}$, where $l_{1}$ and $l_{2}\left(l_{1}<l_{2}\right)$ are expressed by non-zero real numbers. In these cases, the period of conductance oscillations can be very large. For example, if $l_{1}=1.1$ and $l_{2}=3.1$, the asymmetry parameter is $F=\left(l_{2}-l_{1}\right) /\left(l_{2}+l_{1}\right)=1 /(2.1)$ and the period of conductance oscillations is $42 \pi$.

In the following the geometries will be used in which $l_{1}, l_{2}$ are non-zero integers and $l_{1}<l_{2}$. Then the period of conductance oscillation can be much reduced. For example, if $l_{1}=5$ and $l_{2}=7$, the asymmetry parameter is $F=1 / 6$ and the period of conductance oscillations is $12 \pi$. The increase of the parameter $l_{2}$ leads to a decrease of the period.

In the asymmetric ring two types of periodical conductance zeros appear. The first type of zeros, $\eta_{1}=2 m \pi$ $(m \in \mathbb{Z})$, corresponds to the eigenstates of the closed system and the second type, $\eta_{2}=(2 m+1) \pi / F$, is a consequence of the asymmetry [29]. In these points, the interference condition at the right junction can be controlled by the asymmetry parameter $F$.

One can observe in Fig. 3 the asymmetric shape (Fano-type [32]) of the antiresonances in the vicinity of the points $\eta=2 \pi, 4 \pi, 8 \pi$, and $10 \pi$, which is specific for the asymmetric configurations. There are some first type-zeros of the transmission amplitude which are compensated by the poles. For $F=1 / 3$ and $\eta \in[0,12 \pi]$ (solid curve), the compensated zeros are at $\eta=0,6 \pi$ and $12 \pi$ and the conductance has a maximum value in these points. The zeros of the second type are localized at $\eta=3 \pi$ and $\eta=9 \pi$, respectively. For other geometries, the location of these zeros on the $\eta$ axis depends on the asymmetry parameter.

Concerning the dependence of the conductance on the coupling between the leads and the asymmetric ring, we can see from Fig. 3 that with increase of the coupling parameter, the Fano-type antiresonances become more pronounced. For clearly viewing this effect, in Fig. 4 the dimensionless conductance function of the parameter $\eta$ is represented, for asymmetry parameter $F=1 / 3$ and for three values of the coupling parameter: $\varepsilon=1 / 4$ (dotted line), $\varepsilon=4 / 9$ (dashed line) and $\varepsilon=1 / 2$ (solid line). For a fixed value of the asymmetry, the width of the (Fano-type) antiresonance is progressively broadened with increase of the coupling parameter. In addition, the increase of this parameter leads to an increase of the imaginary part for the complex poles of the transmission amplitude.

Close to $\eta=2 \pi$ one such pole is located at $\eta=$ $6.27457-0.172133 \mathrm{i}, \eta=6.20167-0.513457 \mathrm{i}$ and $\eta=$ 


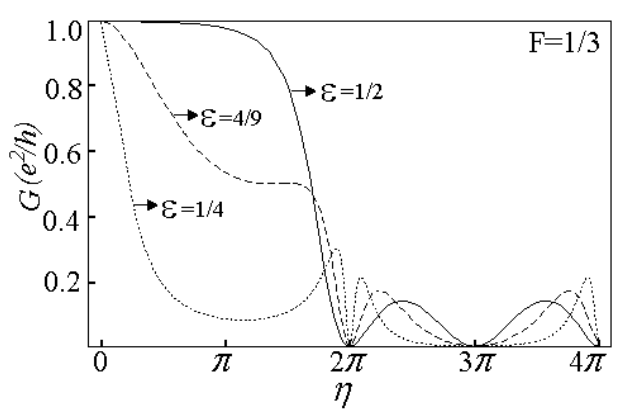

Fig. 4. The dimensionless conductance function of $\eta$ for three values of the coupling parameter: $\varepsilon=1 / 4$ (dotted line), $\varepsilon=4 / 9$ (dashed line) and $\varepsilon=1 / 2$ (solid line); the asymmetry parameter is $F=1 / 3$.

$5.79649-1.03972 \mathrm{i}$, for $\varepsilon=1 / 4,4 / 9$ and $1 / 2$, respectively. The different values of the real part indicate a change of the conductance value at the variation of the coupling parameter.

The locations on the $\eta$ axis for both types of zeros are not affected by this transition from the weak to the strong coupling regime. Therefore, the width of the resonances is changed, depending on the coupling strength.

\subsection{The conductance in the presence of $A B$ flux, but without Rashba SOI}

It is known that the action of the exterior magnetic field leads to the removing of certain conductance zeros, due to the breaking of the time-reversal symmetry (TRS) [33]. The resulting AB flux produces changes in the zero-pole structure and the transmission zeros can be real or can be shifted away from the real energy-axis, depending on the values of the AB phase [33].

For the beginning, there will be investigated only the effect of the magnetic field without the Rashba SOI. For this purpose, two cases are considered: one case in which the AB magnetic flux is an integer or half-integer multiple of the flux quantum and the other, when the AB magnetic flux is off from the integer or half-integer multiples of the flux quantum.

The case in which the magnetic flux is an integer multiple of the flux quantum (the $\mathrm{AB}$ phase is $\Phi_{\mathrm{AB}}=2 m \pi$ ) is equivalent with the case of zero magnetic flux (Fig. 3) for both (symmetric and asymmetric) configurations.

If the magnetic flux is a half-integer multiple of the flux quantum and the $\mathrm{AB}$ phase is $\Phi_{\mathrm{AB}}=(2 m+1) \pi$, the transmission probability for the symmetric ring is zero because of the destructive interference that appears for any value of the ring length.

For the asymmetric ring, the conductance zeros are given by the zeros of the transmission amplitude from Eq. (21):

$$
\exp \left(-\mathrm{i} \frac{(2 m+1) \pi}{2} F\right)\left[\sin \left(\frac{\eta}{2}(1+F)\right)\right.
$$

$$
\left.-\sin \left(\frac{\eta}{2}(1-F)\right)\right]=0 .
$$

The solutions of this equation are

$$
\begin{gathered}
\eta_{1}^{\prime}=(2 m+1) \pi \quad \text { and } \quad \eta_{2}^{\prime}=\frac{2 m \pi}{F}, \\
m=0,1,2,3, \ldots
\end{gathered}
$$

and the relations between the conductance zeros corresponding to $\Phi_{\mathrm{AB}}=2 m \pi$ (or free system) and $\Phi_{\mathrm{AB}}=$ $(2 m+1) \pi$, respectively, are

$$
\eta_{1}^{\prime}=\eta_{1}+\pi \quad \text { and } \quad \eta_{2}^{\prime}=\eta_{2}-\frac{\pi}{F} .
$$

That is, for $\Phi_{\mathrm{AB}}=(2 m+1) \pi$, the first (second) type of transmission amplitude zeros corresponding to the free system are shifted with $\pi(-\pi / F)$ on the $\eta$ axis.

The dependence of the conductance function of $\eta$ in the case when $\Phi_{\mathrm{AB}}=(2 m+1) \pi$ is illustrated in Fig. 5, for different values of the asymmetry parameter and the coupling parameter. Concerning the asymmetry, three different geometries for the $\mathrm{AB}$ ring have been considered, $F=1 / 6, F=1 / 3$ and $F=1 / 2$, with the corresponding angle between the leads $150^{\circ}, 120^{\circ}$ and $90^{\circ}$, respectively. The positions of the transmission amplitude zeros for these asymmetric configurations are indicated in Table I.

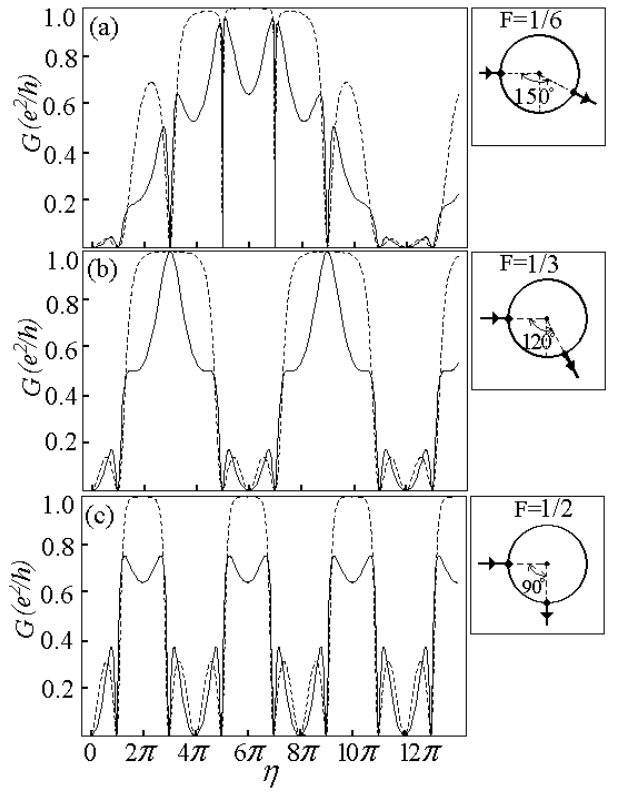

Fig. 5. The dimensionless conductance function of $\eta$ when $\Phi_{\mathrm{AB}}=(2 m+1) \pi$, for $F=1 / 6$ (a), $F=1 / 3$ (b) and $F=1 / 2$ (c). The solid curves correspond to a coupling parameter $\varepsilon=4 / 9$ and the dashed correspond to $\varepsilon=1 / 2$ (at the right of every part there is illustrated the ring with asymmetric contacts; the corresponding angle between the leads is $150^{\circ}$ (a), $120^{\circ}$ (b) and $90^{\circ}$ (c)). 
TABLE I

The transmission amplitude zeros corresponding to the asymmetric configurations in Fig. 5, for $\Phi_{\mathrm{AB}}=2 m \pi$ (free system) and $\Phi_{\mathrm{AB}}=(2 m+1) \pi$.

\begin{tabular}{c|c|c|c|c}
\hline \hline \multirow{2}{*}{ Asymmetry } & \multicolumn{2}{|c|}{$\Phi_{\mathrm{AB}}=2 m \pi$ (free system) } & \multicolumn{2}{c}{$\Phi_{\mathrm{AB}}=(2 m+1) \pi$} \\
\cline { 2 - 5 } & $\eta_{1}$ & $\eta_{2}$ & $\eta_{1}^{\prime}$ & $\eta_{2}^{\prime}$ \\
\hline \multirow{2}{*}{$1 / 6$} & $\begin{array}{c}0,2 \pi, 4 \pi, 6 \pi, 8 \pi, 10 \pi, 12 \pi \\
\text { (compensated: } 0,12 \pi)\end{array}$ & $6 \pi, 18 \pi$ & $\begin{array}{c}\pi, 3 \pi, 5 \pi, \\
7 \pi, 9 \pi, 11 \pi\end{array}$ & $0,12 \pi$ \\
\hline $1 / 3$ & $\begin{array}{c}0,2 \pi, 4 \pi, 6 \pi, 8 \pi, 10 \pi, 12 \pi \\
\text { (compensated: } 0,6 \pi, 12 \pi)\end{array}$ & $3 \pi, 9 \pi$ & $\begin{array}{c}\pi, 3 \pi, 5 \pi, \\
7 \pi, 9 \pi, 11 \pi\end{array}$ & $0,6 \pi, 12 \pi$ \\
\hline $1 / 2$ & $\begin{array}{c}0,2 \pi, 4 \pi, 6 \pi, 8 \pi, 10 \pi, 12 \pi \\
\text { (compensated: } 0,4 \pi, 8 \pi, 12 \pi)\end{array}$ & $2 \pi, 6 \pi, 10 \pi$ & $\begin{array}{c}\pi, 3 \pi, 5 \pi, \\
7 \pi, 9 \pi, 11 \pi\end{array}$ & $0,4 \pi, 8 \pi, 12 \pi$ \\
\hline
\end{tabular}

\section{TABLE II}

Removed and persistent zeros of the transmission amplitude, for the asymmetric configurations in Fig. 6, in the case when the AB flux is not an integer or half integer multiple of the flux quantum.

\begin{tabular}{c|c|c|c}
\hline \hline \multirow{2}{*}{ Asymmetry } & \multirow{2}{*}{$\begin{array}{c}\text { Removed zeros, } \\
\text { of type } \eta_{1}=2 m \pi\end{array}$} & \multicolumn{2}{|c}{ Persistent zeros } \\
\cline { 3 - 4 } & $2 \pi, 4 \pi, 6 \pi, 8 \pi, 10 \pi$ & $\eta_{1}=2 m \pi$ & $\eta_{2}=(2 m+1) \pi / F$ \\
\hline $1 / 6$ & $2 \pi, 4 \pi, 8 \pi, 10 \pi$ & $0,6 \pi, 12 \pi$ & - \\
$1 / 3$ & $2 \pi, 6 \pi, 10 \pi$ & $0,4 \pi, 8 \pi, 12 \pi$ & $3 \pi, 9 \pi$ \\
$1 / 2$ & &
\end{tabular}

One can see from Fig. 5 and Table I that for all selected geometries, the first (second) type zeros for $\Phi_{\mathrm{AB}}=2 m \pi$ (including those which are compensated by the poles of the transmission amplitude) are shifted with $\pi(-\pi / F)$ on the $\eta$ axis for $\Phi_{\mathrm{AB}}=(2 m+1) \pi$. In this way, the compensated zeros for free system (in which the conductance has a maximum value) coincide with the second type zeros for $\Phi_{\mathrm{AB}}=(2 m+1) \pi$ (in which the conductance is zero). Another important aspect is the dependence of the zeros $\eta_{2}^{\prime}$ on the ring asymmetry. Their number increase with the increase of the asymmetry parameter. The conductance zeros are not influenced by the variation of the coupling strength and this leads to a variation of the resonances width.

For viewing the effect of the AB flux which is not an integer or half integer multiple of the flux quantum, in Fig. 6 the dimensionless conductance function of $\eta$ is represented, for $\Phi_{\mathrm{AB}}=2 \pi 0.3$ (solid curves) and for free system ( $\Phi_{\mathrm{AB}}=0$, dashed curves), for comparison. The value of the coupling parameter is set to $\varepsilon=4 / 9$ and the same values of the asymmetry parameter are used, like in Fig. 5.

One may clearly see from Fig. 6 that certain first type-conductance zeros associated to the free system disappear, due to the action of the magnetic field. Without entering in detail, it must be mentioned that the removing of these conductance zeros is equivalent with a shift of the transmission amplitude zeros away from the real-energy axis (see Ref. [33]).

Other conductance zeros of this type persist at the action of the magnetic field, the transmission ampli- tude zeros remaining on the real-energy axis [33]. These persistent conductance zeros coincide with the zeros of the transmission amplitude that are compensated by the corresponding poles in the case of the free system (see Table I and Table II).

For special asymmetries, there are second type-conductance zeros corresponding to the free system that persist. Two of such persistent zeros, for $F=1 / 3$, are located at $\eta=3 \pi$ and $\eta=9 \pi$ (see Fig. 6 and Table II).

In addition, one has to realize that the action of an $\mathrm{AB}$ magnetic flux which is off from the integer or half-integer multiples of the flux quantum, leads to the transformation of the Fano-type antiresonances into simple oscillations. This can be viewed, for example, in Fig. 6 for the geometric configurations with $F=1 / 6$ and $F=1 / 3$. Concerning the lead-ring coupling regime, in the presence of the AB magnetic flux the variation of the coupling strength leads to a changing of the profile of the resonances, the positions of the transmission zeros remaining unaffected.

\subsection{The conductance in the presence of both $A B$ flux and Rashba SOI}

In the presence of the Rashba SOI the time-reversal symmetry is preserved but the spin-parity is broken. For a symmetric AC ring, the effect of SOI leads to the appearance of the conductance zeros. For an asymmetric $\mathrm{AC}$ ring, it is known that the SOI produces the removing of some conductance zeros like in case of the asymmetric $\mathrm{AB}$ ring (depending on the value of the $\mathrm{AC}$ phase) and, 


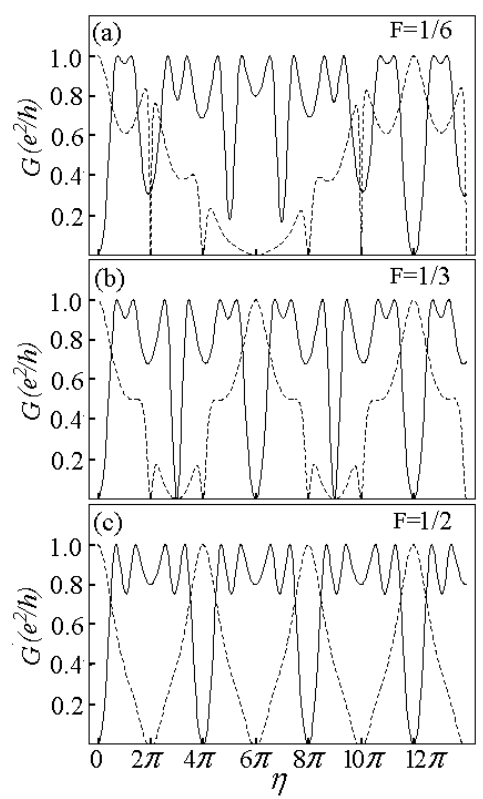

Fig. 6. The dimensionless conductance function of $\eta$ for $F=1 / 6$ (a), $F=1 / 3$ (b) and $F=1 / 2$ (c). The dashed curves correspond to the free system and the solid curves correspond to the case when the $\mathrm{AB}$ flux is not an integer (or half integer) multiple of the flux quantum, $\Phi_{\mathrm{AB}}=2 \pi 0.3$. The coupling parameter is $\varepsilon=4 / 9$.

regarding the symmetry breaking, there is an analogy between the effect of SOI and the effect of the $\mathrm{AB}$ flux for the $1 \mathrm{D}$ ring [29].

So, in the following only an aspect will be investigated, related of the possibility to obtain different spin-up and spin-down conductances in the asymmetric $\mathrm{AB}$ rings, by tuning the $\mathrm{AB}$ and $\mathrm{AC}$ phases. This is based on the fact that the $\mathrm{AB}$ and $\mathrm{AC}$ phases, together with the asymmetry parameter can produce changes in the zero-pole structure.

At some values of these parameters, a compensation of a certain zero with the corresponding pole of the transmission amplitude can be realized for one spin channel. Due to the compensation, one component of the conductance for that spin channel has a finite value. For the other spin channel, that zero-pole pair cannot compensate each other and the conductance is zero.

Suppose that in the presence of $\mathrm{AB}$ flux and Rashba SOI, for one spin species, spin-up for example, the total phase is

$$
\Phi^{\uparrow}=\Phi_{\mathrm{AB}}+\Phi_{\mathrm{AC}}^{\uparrow}=2 m \pi, \quad \text { with } m=1,2,3, \ldots
$$

This may be satisfied for certain values of $A B$ phase and dimensionless Rashba parameter. If one takes $\Phi_{\mathrm{AB}}=$ $2 q \pi$, with $0<q<m$ (the parameter $q$ must be non-integer (half integer) to obtain different spin-up and spin-down components of the conductance), the dimensionless Rashba parameter is given by the expression

$$
\xi=\sqrt{(2(m-q)+1)^{2}-1} .
$$

In this case, the total phase for the spin-down species reads

$$
\Phi^{\downarrow}=2 \pi(2 q-m-1) .
$$

One has to realize that, for such values of the phases $\Phi^{\uparrow}$ and $\Phi^{\downarrow}$, in the persistent zeros of the spin-up transmission amplitude which are compensated by the corresponding poles in the case of free system, the spin-up conductance has a finite (maximum) value and the spin-down conductance is zero.

In Fig. 7 the spin-up and spin-down conductances function of $\eta$ are displayed, for the asymmetry parameter $F=1 / 3$ and for different values of the coupling parameter. The $\mathrm{AB}$ phase is set to $\Phi_{\mathrm{AB}} / 2 \pi=0.08$ and the dimensionless Rashba parameter is $\xi=2.65812$, which satisfies the condition (28) for $m=1$.

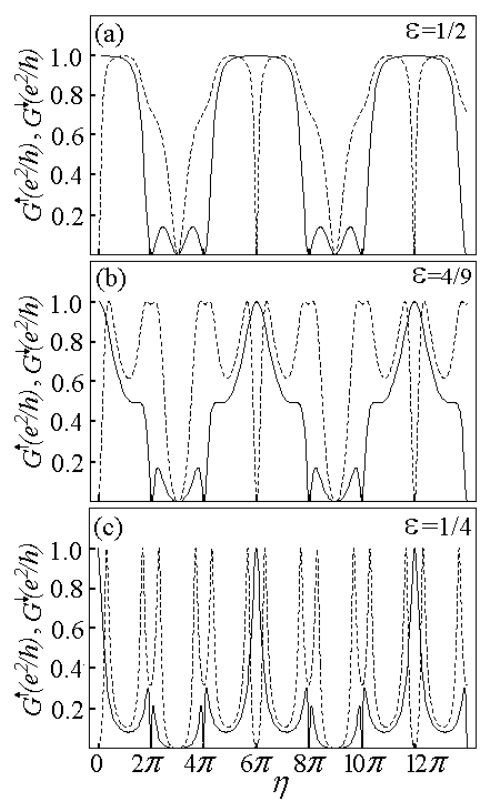

Fig. 7. The spin-up (solid line) and spin-down (dashed line) conductances function of $\eta$, for $F=1 / 3$ and $\varepsilon=1 / 2$ (a), $\varepsilon=4 / 9$ (b), $\varepsilon=1 / 4$ (c). The AB phase is $\Phi_{\mathrm{AB}} / 2 \pi=0.08$ and the dimensionless Rashba parameter is $\xi=2.65812$.

For this configuration, if $\eta=2 m \pi$ ( $m$ - integer $)$ except the persistent zeros $\eta=0,6 \pi$ and $12 \pi$, the transmission probability in the spin-up channel is zero (solid curves in Fig. 7) and the transmission probability in the spin-down channel has a non-zero value that depends on the coupling parameter (dashed curves in Fig. 7).

At the persistent zeros (compensated by the poles in the case of free system) localized in the points $\eta=0,6 \pi$ and $12 \pi$, the $\mathrm{AB}$ ring is totally opaque for the spin-down electrons and transparent for the spin-up electrons, for any value of the coupling parameter. At the persistent zeros localized in the points $\eta=3 \pi$ and $9 \pi$ the device is in a totally reflective state. For another values of the 
asymmetry parameter, the location and the number of the persistent zeros are changed. At the variation of the coupling parameter, the behavior of the conductance is the same like in the precedent cases.

So, tuning the $\mathrm{AB}$ and $\mathrm{AC}$ phases, the asymmetric $\mathrm{AB}$ ring can act like a spin filter device and the efficiency of the filtering process can be modified by changing the coupling parameter and the asymmetry parameter.

It must be pointed that for a strong spin-orbit coupling, the inter-subband mixing becomes important and has a destructive effect on the filtration. One way to minimize this subband mixing is to adjust the transverse width of the $\mathrm{AB}$ rings in order to be very narrow compared with their length (in practice, the real rings are not quite strictly one-dimensional). In this case the energy levels spacing resulting from the transversal confinement become large. This can be verified experimentally using e.g. the split-gate techniques [2, 34].

Moreover, it is known that in general, for a quantum waveguide device, the number of the propagating channels is $N=\operatorname{Int}\left(k_{\mathrm{F}} w / \pi\right)$, in which $k_{\mathrm{F}}$ is the Fermi wave number and $w$ is the width of the waveguide [34]. Therefore, using selected Fermi energies of the incident electrons through the $\mathrm{AB}$ ring and an adequate tuning of the transverse width, one can reduce the number of channels (subbands). So, the effect of the inter-subband mixing is reduced and a better spin filtering can be obtained.

On the other hand, the inter-subband mixing can be neglected if the width of the confining potential well obeys the condition $w_{\mathrm{C}} \ll \hbar^{2} / \alpha_{R} m^{*}$ [35]. For an InGaAs based $\mathrm{AB}$ ring with the radius $R=250 \mathrm{~nm}$, $m^{*}=0.023 m_{\mathrm{e}}$ (where $m_{\mathrm{e}}$ is the free electron mass) and a Rashba SOI coupling constant $\alpha_{R}=2 \times 10^{-11} \mathrm{eV} \mathrm{m}$ $[7,15]$, this corresponds to a dimensionless constant like $\xi \cong 3.0142$. In this case, the above condition yields $w_{\mathrm{C}} \ll 0.166 \mu \mathrm{m}$, which can be verified experimentally.

\section{Conclusions}

In summary, the transmission amplitude for the asymmetric AB ring with the Rashba SOI was derived analytically in the adiabatic limit, using the transfer matrix formalism. The conductance such as given by the Landauer formalism, depends on the electron energy, the asymmetry and coupling parameters and on the total phase. The transition from weak to strong coupling leads to a progressive broadening of the width of the Fano-type antiresonances.

For the asymmetric ring in the presence of the AB magnetic flux, the behaviour of the transmission amplitude zeros depends on the values of the $\mathrm{AB}$ phase. The case when $\Phi_{\mathrm{AB}}=2 m \pi\left(\Phi / \Phi_{0}\right.$ is an integer $)$ is similar to the free system while in the case $\Phi_{\mathrm{AB}}=(2 m+1) \pi\left(\Phi / \Phi_{0}\right.$ is a half-integer) the conductance zeros are only shifted on the $\eta$ axis.

In the case when the $\mathrm{AB}$ flux is not an integer or half integer multiple of the flux quantum, some conductance zeros are removed, due to the breaking of the time-reversal symmetry by the magnetic field, whereas some other conductance zeros persist. Moreover, we found that the Fano-type resonances are changed into ordinary oscillations.

For the asymmetric ring in the presence of both $\mathrm{AB}$ flux and Rashba SOI, a zero-pole compensation can be done in one spin channel to obtain a finite conductance, by an adequate adjusting of the $\mathrm{AB}$ and $\mathrm{AC}$ phases, in which case the conductance in the other channel is zero. This opens the way to use the AB asymmetric ring like a spin-filter device in which the electron transmission is sensitive to the modification of the lead-ring coupling strength as well as to the asymmetry parameter.

\section{Acknowledgments}

The author would like to thank Prof. Dr. Erhardt Papp for reading the manuscript and for helpful comments and suggestions.

\section{References}

[1] I. Žutić, J. Fabian, S. Das Sarma, Rev. Mod. Phys. 76, 323 (2004).

[2] Semiconductor Spintronics and Quantum Computation, Eds. D.D. Awschalom, N. Samarath, D. Loss, Springer-Verlag, Berlin 2002.

[3] Spin-Orbit Coupling Effects in Two-Dimensional Electron and Hole Systems, R. Winkler, Springer, New York 2003.

[4] E.I. Rashba, Sov. Phys. Solid State 2, 1109 (1960).

[5] G. Dresselhaus, Phys. Rev. 100, 580 (1955).

[6] B. Das, D.C. Miller, S. Datta, R. Reifenberg, W.P. Hong, P.K. Bhattacharya, J. Singh, M. Jaffe, Phys. Rev. B 39, 1411 (1989).

[7] J. Nitta, T. Akazaki, H. Takayanagi, T. Enoki, Phys. Rev. Lett. 78, 1335 (1997).

[8] D. Grundler, Phys. Rev. Lett. 84, 6074 (2000).

[9] G. Lommer, F. Malcher, U. Rossler, Phys. Rev. Lett. 60, 728 (1988).

[10] Y. Aharonov, D. Bohm, Phys. Rev. 115, 485 (1959).

[11] Y. Aharonov, A. Casher, Phys. Rev. Lett. 53, 319 (1984).

[12] J. Splettstoesser, M. Governale, U. Zülicke, Phys. Rev. B 68, 165341 (2003).

[13] J. Nitta, T. Koga, F.E. Meijer, Physica E 18, 143 (2003).

[14] D. Frustaglia, K. Richter, Phys. Rev. B 69, 235310 (2004).

[15] B. Molnár, F.M. Peeters, P. Vasilopoulos, Phys. Rev. B 69, 155335 (2004).

[16] R. Citro, F. Romeo, M. Marinaro, Phys. Rev. B 74, 115329 (2006).

[17] N.T. Bagraev, N.G. Galkin, W. Gehlhoff, L.E. Klyachkin, A.M. Malyarenko, I.A. Shelykh, Physica B 378-380, 894 (2006).

[18] S. Pedersen, A.E. Hansen, A. Kristensen, C.B. Sorensen, P.E. Lindelof, Phys. Rev. B 61, 5457 (2000). 
[19] U.F. Keyser, S. Borck, R.J. Haug, M. Bichler, G. Abstreiter, W. Wegscheider, Semicond. Sci. Technol. 17, L22 (2002).

[20] M. Büttiker, in: SQUID'85 - Superconducting Quantum Interference Devices and their Applications, Eds. H.D. Haklbohm, H. Lubbig, Walter de Gruyter, Berlin 1985, p. 529.

[21] E.R. Hedin, R.M. Cosby, A M. Satanin, Y.S. Joe, J. Appl. Phys. 97, 063712 (2005).

[22] C. Benjamin, A.M. Jayannavar, Phys. Rev. B 65, 153309 (2002).

[23] K. Wakabayashi, Phys. Rev. B 64, 125428 (2001).

[24] S.S. Buchholz, S. Fischer, U. Kunze, M. Bell, D. Reuter, A.D. Wieck, Physica E 42, 1099 (2010).

[25] M. Büttiker, I. Imry, M.Ya. Azbel, Phys. Rev. A 30, 1982 (1984).

[26] D.M. Baltateanu, Phys. Lett. A 375, 2952 (2011).
[27] M. Büttiker, Y. Imry, R. Landauer, S. Pinhas, Phys. Rev. B 31, 6207 (1985).

[28] F.E. Meijer, A.F. Morpurgo, T.M. Klapwijk, Phys. Rev. B 66, 33107 (2002).

[29] U. Aeberhard, K. Wakabayashi, M. Sigrist, Phys. Rev. B 72, 075328 (2005).

[30] S. Griffith, Trans. Faraday Soc. 49, 650 (1953); J.B. Xia, Phys. Rev. B 45, 3593 (1992).

[31] W. Porod, Z. Shao, C.S. Lent, Phys. Rev. B 48, R8495 (1993).

[32] U. Fano, Phys. Rev. 124, 1866 (1961).

[33] T.S. Kim, S.Y. Cho, C.K. Kim, C.M. Ryu, Phys. Rev. B 65, 245307 (2002).

[34] H. van Houten, C.W.J. Beenakker, Physics Today 49, 22 (1996).

[35] S. Datta, B. Das, Appl. Phys. Lett. 56, 665 (1990). 